\title{
Electrical stimulation-assisted rowing exercise in spinal cord injured people. A pilot study
}

\author{
J J Laskin BSc, ${ }^{1}$ E A Ashley, ${ }^{3 *}$ L M Olenik BSc, ${ }^{1}$ R Burnham MD FRCP(C) ${ }^{1}$ \\ D C Cumming MB ChB FRCSC,${ }^{2} \mathrm{R}$ D Steadward PhD,${ }^{1}$ G D Wheeler $\mathrm{PhD}^{1 \dagger}$ \\ ${ }^{1}$ The Rick Hansen Centre, ${ }^{2}$ Department of Obstetrics and Gynaecology, Division of \\ Endocrinology, University of Alberta, Edmonton, Canada; ${ }^{3}$ Institute of Physiology, \\ University of Glasgow, Scotland.
}

\begin{abstract}
Recently a FES (functional electrical stimulation)-assisted rowing machine was developed to enhance cardiovascular training in people with spinal cord injuries. The machine was assessed in terms of its efficacy as a training tool. Six patients who were quadriplegic (C6-T1) and 2 who were paraplegic (T3-6) completed a series of three tests in succession: (1) leg stimulation only (quadriceps and hamstring groups)-'Stim', (2) arm row only-'Row' and (3) simultaneous row and stimulation-' $R$ \& $S$ '. Measurements recorded included oxygen uptake (VO2), minute ventilation (Ve), respiratory exchange ratio (RER), heart rate (HR) and blood pressure (BP). In addition, 6 out of the 8 subjects took part in a qualitative assessment comprising a guided interview exploring the subject's perception of the machine and test.

Significant increases in VO2 were demonstrated between the three tests with $\mathrm{R} \& \mathrm{~S}$ producing mean steady-state values of $16.34 \mathrm{~nm}( \pm 0.74) \mathrm{ml} / \mathrm{kg} / \mathrm{min}(83 \%$ of maximum). These values represented a $12 \%$ increase over Row alone. Of interest was the qualitative assessment which revealed that subjects perceived $\mathrm{R}$ $\& \mathrm{~S}$ to be easier than Row despite the higher levels of VO2 observed. The results suggest that the rowing machine represents a potentially valuable hybrid training device that may significantly reduce risk factors for cardiovascular disease and improve the quality of life of people with SCI.
\end{abstract}
Keywords: functional electrical stimulation; spinal cord injured; hybrid rowing machine; qualitative assessment.

\section{Introduction}

In 1986, it was reported that renal complications no longer accounted for the greatest proportion of morbidity and mortality in people with spinal cord injuries (SCI) and that this position was now held by cardiovascular disease. ${ }^{1}$ Since then much research in this field has centered around designing appropriate devices to meet the need for aerobic training and subject satisfaction in attaining cardiovascular system (CVS) benefit.

\footnotetext{
*Supported by the Faculty of Medicine (Department of Obstetrics and Gynaecology) and the Rick Hansen Centre, University of Alberta, Edmonton, Canada. ${ }^{\dagger}$ Correspondence: The Rick Hansen Centre, W1-67 Van Vliet Centre, University of Alberta, Edmonton T6G 2H9, Canada.
}

In this regard, two distinct approaches can be identified. The most accessible approach was to make use of the muscle mass under voluntary control in programs such as arm crank ergometry (ACE) and weight training. These programs have been shown to be beneficial, promoting increases in maximal oxygen uptake (VO2max), decreases in submaximal heart rate (HR), and decreases in systolic blood pressure and percent body fat. ${ }^{2}$ Unfortunately, it seems that these programs are limited in their ability to produce marked CVS training effects. There are several reasons for this: the small amount of muscle mass available, ${ }^{3}$ the specificity of the circulatory adaptations to the trained muscle, ${ }^{4}$ and the effects of circulatory hypokinesis (particularly in the 
lower limb, causing venous pooling due to loss of the skeletal muscle venous pump).

In the attempt to improve CVS training programs, increasing attention is being paid to the technique of functional electrical stimulation (FES). By applying electrical stimulation to the paralyzed lower limb musculature of people with spinal cord injuries, a greater muscle mass is made available and, theoretically, higher central CVS adaptation is possible. Several studies of FES-assisted exercise have shown not only improvement in CVS conditioning but also increased bone mass, ${ }^{5}$ a reduction of decubitus ulcers, ${ }^{6}$ and increased muscle mass. $^{7}$

It would follow that the greatest potential for central CVS conditioning lies in the combination of lower limb (FES-assisted) exercise with voluntary upper limb exercise. There has been some research examining the physiological effects of 'hybrid' training regimes. For example, Edwards and Marsolais $(1990)^{8}$ showed increases in VO2 during combined ACE and leg cycle exercise (LCE) over ACE or LCE alone. Davis et $a l^{9}$ showed that concurrent FES-induced contraction of the lower limb muscles increases venous return and aids central CVS control during upper body submaximal exercise. More recently, Hooker et al ${ }^{10}$ documented increases in VO2 by $54 \%$ and cardiac output by $33-47 \%$ on combined subpeak ACE and LCE when compared with either exercise mode alone. These studies would seem to support the use of hybrid exercise as an effective tool for central CVS conditioning.

An FES-assisted rowing ergometer 'ROWSTIM' ${ }^{\circledR}$ has been developed at the Rick Hansen Centre, University of Alberta, Edmonton, Alberta, in association with Concept II Inc, Morrissville, Vermont (Fig 1).

The purpose of this study was to test the efficacy of this system in terms of (a) subject reaction and (b) cardiopulmonary training effects.

\section{Methods}

Subjects and methods

Six quadriplegic (C6-T1) and 2 paraplegic
(T3-6) subjects gave informed consent to participate in this study, which was approved by an institutional ethics review body.

In this design, the subject uses FES on both the quadriceps and hamstring groups of muscles and adjusts the on-off cadence and level of stimulation in order to propel him/herself backwards and forwards. The design incorporates a bucket chair for trunk stabilization, leg supports for biomechanical efficiency, and an elastic 'leash' which acts as a brake on extension and biases the movement towards flexion (to aid the generally weaker hamstring muscles). Stimulation is achieved by use of a hand-held stimulator ('Quadstim', Biomech Designs Ltd) with a specially designed adaptor to allow the cadence to be altered. The design will eventually include a quick-release, moulded grip to better facilitate the use of this device by people with quadriplegia.

\section{Protocol}

The test protocol comprised three parts: (1) leg stimulation only ('Stim'), (2) arm rowing only ('Row') and (3) arm rowing and leg stimulation together ('R \& S'). The order of the tests was randomized to control for a cumulative fatigue effect. Exercise was for 10 minutes and a rest period corresponding to $1: 3$ was allowed for values to return to baseline.

On arrival at the lab, subjects were transferred into the 'ROWSTIM' apparatus. A total of eight electrodes (Chattanooga Corp) were placed over motor points and musculotendinous junctions of the quadriceps and hamstring groups of muscles. Metabolic variables were measured by expired air analysis. A metabolic cart (MMC Horizon, Sensormedics, CA) produced 15 second values for oxygen consumption (VO2), minute ventilation (Ve) and respiratory exchange ratio (RER). These values were then averaged for 1-minute periods over the 40-minute protocol. A PE sport-tester (Model 3000) monitored heart rate (HR) continuously and blood pressure was measured by auscultation immediately before and after every test.

On a separate occasion, VO2 max was 

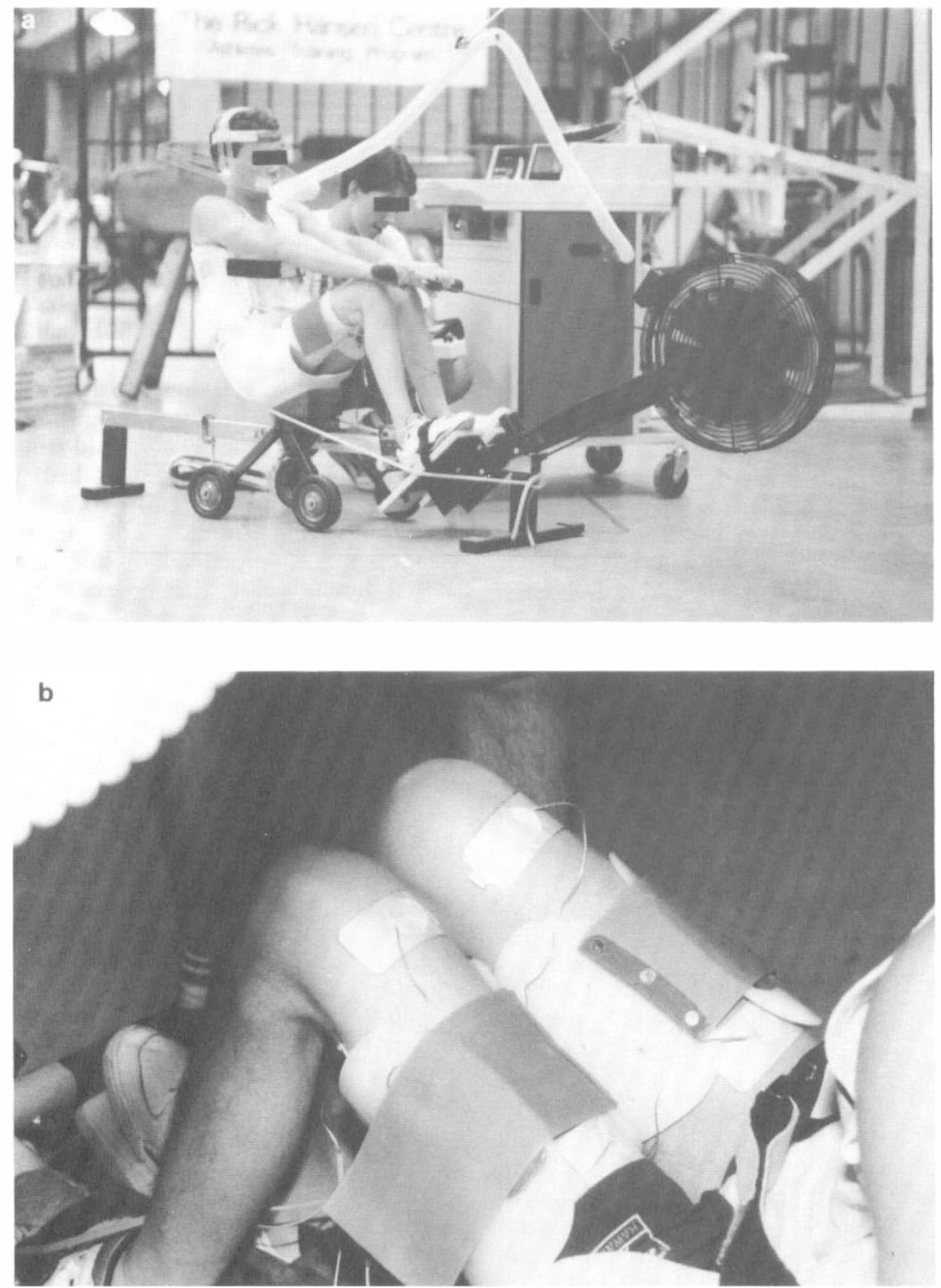

Figure 1 (a) The ROWSTIM apparatus, (b) ROWSTIM leg stabilization procedure.

assessed by an incremental arm ergometry method. The test protocol required the subjects to maintain a cadence of $50 \mathrm{rpm}$. Initial power output for a 2-minute warm-up was set at $10 \mathrm{~W}$ and was increased by $10 \mathrm{~W}$ every 1 or 2 minutes thereafter depending on the musculature of the subject. The test was considered to be over if the cadence fell below $50 \mathrm{rpm}$ or if the subject reached exhaustion. 
The qualitative study consisted of a guided interview conducted at the end of the test with 6 of the 8 subjects. Two subjects did not wish to be recorded. Each interview explored three topics: perception of physical response to test, perception of ROWSTIM as a training device and motivation to participate in FES research.

Following transcription of the interviews, relevant quotes and comments were identified through descriptive analysis. Further interpretive analysis was completed to identify common trends and relationships. Connections between quantitative and qualitative responses were independently determined by three analysts looking at the data separately.

\section{Data analysis}

Values are reported for Rest (mean value for final 5 minutes); Stim (peak); Row (peak); and R \& S (peak). Cardiovascular system values represent immediate posttest measurements. Values for $\mathrm{VO} 2$ are reported: in $1 / \mathrm{min}$ (absolute); in $\mathrm{ml} / \mathrm{kg} / \mathrm{min}$ (relative); and as \% max (percentage). Blood pressure measurements are reported as mean arterial pressure (MAP) (ie diastolic $+1 / 3$ pulse pressure).

An analysis of variance with repeated measures, was carried out on all the measured variables. Post-hoc Scheffe multiple comparisons were computed after a significant main effect. Data are presented as means \pm standard error (SE) and a significance level was set at $p<0.05$.

\section{Results}

Subject details are reported in Table I. Resting and cardiovascular variables are reported in Table II. As there were no significant differences in the cardiovascular responses within the subject group, the data was pooled. Values for absolute, relative and percent $\mathrm{VO} 2$ all showed significant increases from Rest to Stim to Row to $\mathrm{R} \& \mathrm{~S}$. The percentage of $\mathrm{VO} 2 \max$ increased by 46.1 from Stim to $R$ \& $S$ and 11.9 from Row to R \& S (Fig 2).

Values for Ve (Fig 3) also significantly increased from Rest to Stim, from Rest to Row and from Rest to R \& S though the increase from Row to $\mathrm{R} \& \mathrm{~S}$ was not

Table I Subject information

Subject Age (yrs) Gender Level Time since injury (yrs)

\begin{tabular}{|c|c|c|c|c|}
\hline 1 & 25 & $\mathrm{M}$ & T3-4 & 10 \\
\hline 2 & 26 & $\mathrm{~F}$ & C6-7 & 8 \\
\hline 3 & 29 & M & C6-7 & 12 \\
\hline 4 & 24 & M & C7 & 2 \\
\hline 5 & 25 & $\mathrm{M}$ & C6-7 & 7 \\
\hline 6 & 37 & $\mathrm{M}$ & T5-6 & 20 \\
\hline 7 & 29 & M & C7-T1 & 2 \\
\hline 8 & 28 & $\mathrm{M}$ & C6 & 4 \\
\hline
\end{tabular}

Table II A repeated measures ANOVA for all variables (see text for details)

\begin{tabular}{|c|c|c|c|c|c|c|c|c|c|}
\hline Var & Units & REST & SE & STIM & SE & ROW & SE & $R \& S$ & SE \\
\hline$\dot{\mathrm{V}} \mathrm{O}_{2}$ & $1 / \min$ & $0.229^{\mathrm{a}, \mathrm{b}, \mathrm{c}}$ & 0.018 & $0.464^{\mathrm{b}, \mathrm{c}, \mathrm{d}}$ & 0.025 & $0.825^{\mathrm{a}, \mathrm{c}, \mathrm{d}}$ & 0.060 & $1.020^{\mathrm{a}, \mathrm{b}, \mathrm{d}}$ & 0.052 \\
\hline$\dot{\mathrm{V}} \mathrm{O}_{2}$ & $\mathrm{ml} / \mathrm{kg} / \mathrm{min}$ & $3.64^{\mathrm{a}, \mathrm{b}, \mathrm{c}}$ & 0.20 & $7.49^{b, c, d}$ & 0.46 & $13.27^{\mathrm{a}, \mathrm{c}, \mathrm{d}}$ & 1.01 & $16.34^{\mathrm{a}, \mathrm{b}, \mathrm{d}}$ & 0.74 \\
\hline$\dot{\mathrm{V}} \mathrm{O}_{2}$ & $\% \max$ & $18.1^{\mathrm{a}, \mathrm{b}, \mathrm{c}}$ & 0.9 & $36.8^{\mathrm{b}, \mathrm{c}, \mathrm{d}}$ & 1.5 & $71.0^{\mathrm{a}, \mathrm{c}, \mathrm{d}}$ & 3.3 & $82.9^{\mathrm{a}, \mathrm{b}, \mathrm{d}}$ & 3.4 \\
\hline$\dot{\mathrm{V}}_{\mathrm{e}}$ & $1 / \mathrm{min}$ & $8.9^{\mathrm{a}, \mathrm{b}, \mathrm{c}}$ & 0.4 & $16.7^{b, c, d}$ & 0.8 & $32.9^{\mathrm{a}, \mathrm{d}}$ & 2.6 & $34.4^{\mathrm{a}, \mathrm{d}}$ & 3.1 \\
\hline RER & & $0.77^{\mathrm{a}, \mathrm{b}, \mathrm{c}}$ & 0.03 & $0.94^{\mathrm{d}}$ & 0.03 & $0.99^{d}$ & 0.03 & $0.96^{\mathrm{d}}$ & 0.02 \\
\hline HR & bpm & $69^{b, c}$ & 7 & $71^{b, c}$ & 9 & $112^{\mathrm{a}, \mathrm{d}}$ & 10 & $105^{\mathrm{a}, \mathrm{d}}$ & 8 \\
\hline MAP & $\mathrm{mmHg}$ & $82.2^{c}$ & 2.0 & $95.4^{\mathrm{b}}$ & 1.0 & $76.7^{\mathrm{a}, \mathrm{c}}$ & 3.9 & $96.5^{b, d}$ & 4.0 \\
\hline
\end{tabular}

$\dot{\mathrm{V}} \mathrm{O}_{2} \max (\mathrm{ml} / \mathrm{kg} / \mathrm{min})$ 19.49; SE 2.84 .

Body mass (kg) 63.2; SE 4.2.

${ }^{a} p<0.05$ from leg stimulation only (STIM).

b $p<0.05$ from arm row only (ROW).

c $p<0.05$ from leg stimulation and arm row $(\mathrm{R} \& \mathrm{~S})$.

${ }^{\mathrm{d}} p<0.05$ from Rest. 


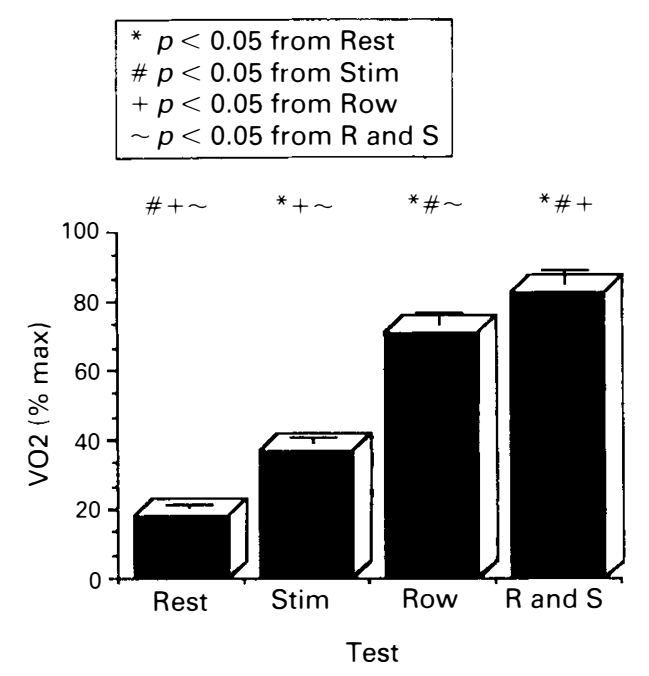

Figure 2 Oxygen uptake expressed as a \% of maximum $( \pm S E)$ for the three tests.

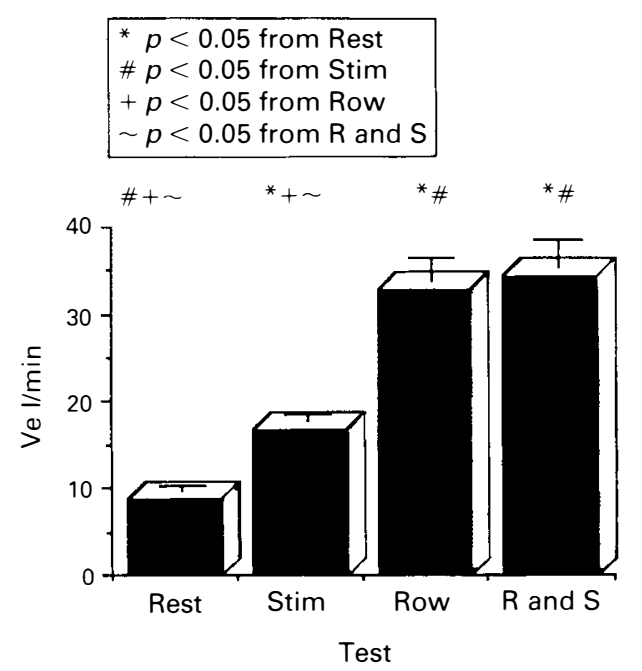

Figure 3 Minute ventilation for the three tests $( \pm \mathrm{SE})$.

significant. The RER (Fig 4) was significantly increased under all three conditions from rest, but was in fact lower for $\mathrm{R} \& \mathrm{~S}$ than for Row.

Heart rate (Fig 5) was not significantly changed from Rest to Stim but was significantly higher from Rest to Row and Rest to $\mathrm{R} \& \mathrm{~S}$, as well as from Stim to Row and Stim to R \& S. However, it should be noted

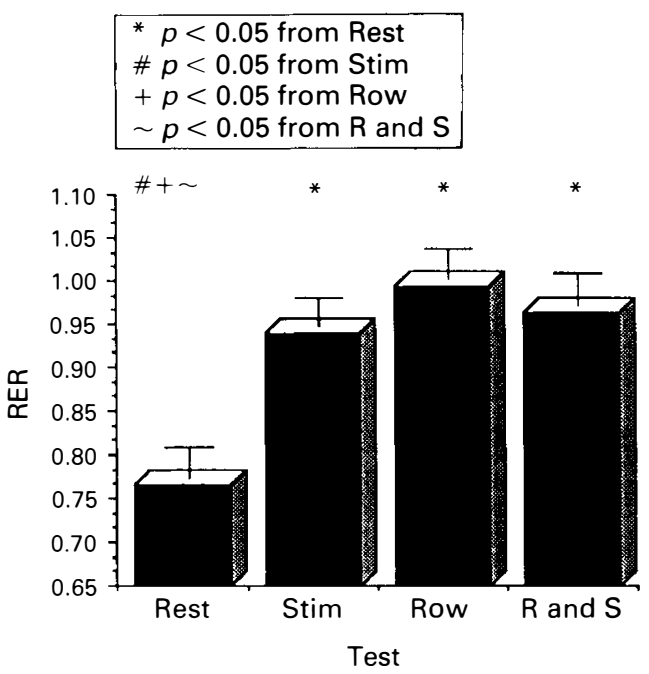

Figure 4 The RER for the three tests $( \pm S E)$.

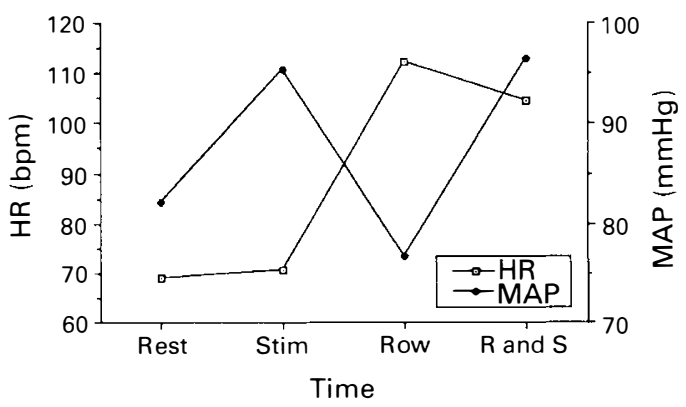

Figure 5 Cardiovascular variables measured immediately post each test.

that the value for $\mathrm{R} \& \mathrm{~S}$ was lower than the value for Row alone.

Mean arterial pressure (Fig 5) was significantly increased from Rest to R \& S and was significantly decreased from Stim to Row, while Row to $\mathrm{R} \& \mathrm{~S}$ showed a significant increase.

The qualitative assessment revealed that 5 out of 6 subjects perceived Row to be harder than R \& S. Five out of 6 subjects did not understand their physiological responses to FES and rowing while only one subject asked questions about his physiological state in general. With regard to motivation, 4 subjects stated that they participated because of the possibility of helping someone else, while 3 participated as a means to improve their own physical health. 
Three subjects volunteered improvements which they thought could be made to the design to make it more accessible and to make the mechanical action smoother.

\section{Discussion}

The increased oxygen consumption associated with simultaneous rowing and leg stimulation is an important finding in the attempt to develop a form of aerobic training exercise that will reduce risk factors for cardiovascular disease in people with spinal cord injuries. The data suggested that the aerobic capacity of people with SCI is primarily limited by the amount of muscle mass under voluntary control rather than by the cardiovascular system's restraints. Since excitation of additional muscle mass results in increased oxygen consumption it is not surprising that one subject rowed at $125 \%$ of his VO2 max (as assessed by arm ergometry). This is consistent with the work of Edwards and Marsolais. ${ }^{8}$ Their subjects had low lesion levels (T6-T11) which are associ- ated with a much larger amount of muscle mass under voluntary control. (The negative correlation between injury level and VO2 max is well established. ${ }^{11}$ ) In addition, they used intramusclular electrodes to excite a much larger amount of lower limb musculature including gluteus maximus muscles of the lower leg as well as the thigh muscles. This may explain the high values of $\mathrm{VO} 2$ exhibited. A comparison of the present study with several others is shown in Figure 6 . It can be seen that VO2 levels achieved with ROWSTIM compare very favourably to levels achieved with subjects with similar disabilities for knee extension, ${ }^{12}$ LCE (using ERGYS I cycle, Therapeutic Technologies Inc) ${ }^{13} \mathrm{ACE},{ }^{11}$ and hybrid exercise comprising $\mathrm{ACE}$ and $\mathrm{LCE} .^{10}$ In addition, it is encouraging that a new device, despite the many modifications and improvements still possible, is capable of eliciting such high degrees of $\mathrm{O} 2$ uptake.

As expected, values for $\mathrm{Ve}$ increased in line with increases in VO2. Furthermore, we noted that the ratio $\mathrm{Ve}: \mathrm{VO} 2$ increased

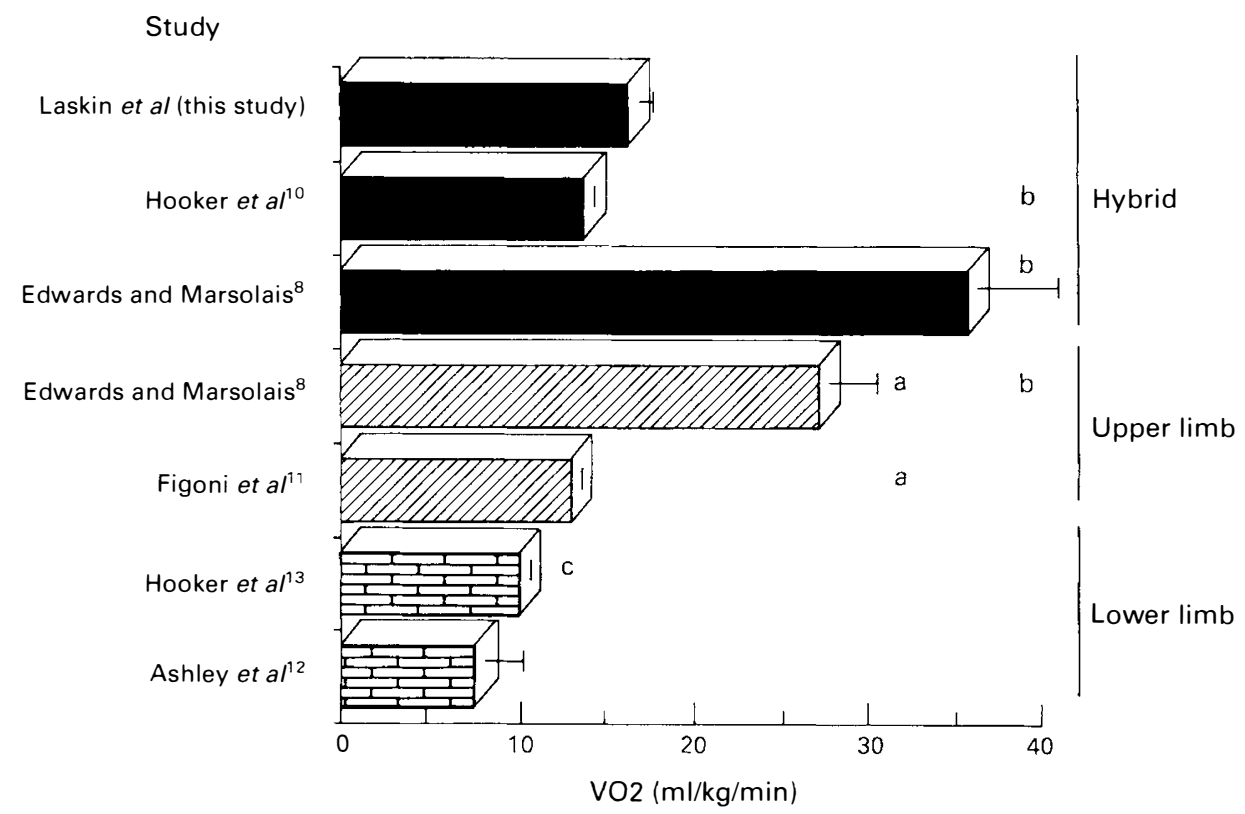

Figure 6 A multistudy comparison of values for VO2. (a) Values for maximal exercise. (b) Standard errors calculated separately. Conversions to $\mathrm{ml} / \mathrm{kg} / \mathrm{min}$ carried out using mean body weight measurements reported in the original papers. (c) Value taken from graph in the original paper. 
linearly throughout the exercise. This suggests that the mechanics of rowing do not impede respiratory efficiency.

The RER significantly increased from rest to all three test conditions but not between the tests. For several reasons, ${ }^{12}$ the RER may be expected to be higher during stimulation than during Row, but in fact it was higher during Row (though not significantly). However, this may be unique to this particular investigation.

The HR during the Stim test fell dramatically; this was probably a baroreceptormediated response to the stimulation. During Row, however, the HR response mirrored an able bodied HR during exercise. This increase in HR could partly explain the greater perceived difficultly of Row versus R \& S. ${ }^{14}$

In comparison, the MAP response dropped from a resting level immediately post Row, perhaps indicating a degree of lower limb venous pooling or lack of compensatory vasoconstriction in nonexercising body parts. Of further interest, the MAP values for $R \& S$ were only slightly higher than those for Stim (whereas HR was significantly higher) yet both MAPs were significantly higher than the MAP for Row. Considering the fact that more muscle mass is being accessed and VO2 is higher, this would be an unexpected finding except for the ensuing discussion of autonomic dysreflexia.

It is possible that the CVS variables showed a response reminiscent of FESinduced autonomic dysreflexia (for a full account see Ashley et al ${ }^{12}$ ). It seems that the extra musculature used for $\mathrm{R} \& \mathrm{~S}$ serves to decrease the sympathetically-mediated vasoconstriction of the dysreflexic response to the extent that for a much higher VO2, the MAP is only slightly raised. This finding suggests that the nutritional requirement of the working muscle is able to overcome the neurally-imposed vasoconstriction. This is consistent with the exercise response in the able bodied population. ${ }^{15}$

Despite the likelihood of their dysreflexic state, subject perceptions indicated that they were unaware of their physical state. As previously stated, only one subject questioned the lower than expected HR. Of the remaining subjects none had questions related to their physiological responses to FES, despite being made aware of results previous to the interview. This could be related to the lack of motivation illustrated by half of the subjects who stated that they were not interested in FES personally, but had participated in order to help 'other' people. Furthermore, in spite of the fact that physiological responses showed that they were working at a higher percentage of maximum during $\mathrm{R} \& \mathrm{~S}, 5$ out of 6 subjects interviewed felt that $\mathrm{R} \& \mathrm{~S}$ was less difficult and therefore less of a workout than Row. This implies that subjects would be able to maintain a higher workload, with greater comfort, for longer periods of time.

The researchers suggest that rowing represents a better hybrid training activity than simultaneous ACE and LCE. The coordination of upper and lower limbs would seem to be easier and more natural in the action of rowing. This is supported by the qualitative findings in that subjects perceived Row to be biomechanically awkward in comparison to $\mathrm{R} \& \mathrm{~S}$. Moreover, the fact that the ROWSTIM machine is a minimally adapted version of a machine for able bodied people makes it easier to manufacture and perhaps more accessible for a broader range of subjects.

There are many advantages of hybrid exercise training regimes. The use of lower limb contractions has been shown to aid venous return via the skeletal muscle venous pump. ${ }^{7}$ This would result in a greater end-diastolic volume and through the Frank-Starling relationship higher contractility and ultimately increased stroke volume and cardiac output. The implications of this for cardiac training are important and well documented. ${ }^{16}$

In addition, spreading the load over a much greater muscle mass, as is the case with hybrid exercise, should result in a delayed time to fatigue. This, combined with higher levels of $\mathrm{VO} 2$ and, we suggest, higher levels of cardiac output (as demonstrated by Hooker and coworkers ${ }^{9}$ ) could result in potentially increased CVS training effects in the SCI population.

In summary, the ROWSTIM device developed at the Rick Hansen Centre has been 
shown capable of eliciting mean, steadystate values for $\mathrm{VO} 2$ of over $80 \%$ of $\mathrm{ACE}$ maximum. It has been shown to be well received by SCI subjects who perceive the addition of leg stimulation to result in an easier workout, despite this inducing higher levels of VO2. Consequently, it is felt that this device represents great potential for cardiovascular training of people with SCI, and therefore the reduction of risk factors for cardiovascular disease.

\section{Acknowledgements}

The authors would like to thank the subjects for their patience, cooperation and good humour in completing this research and Heather Wilkins for her assistance with data collection. This research was supported in part by the Recreation Parks and Wildlife Foundation, Alberta Lotteries the Alberta Paraplegic Foundation, and the Harvey Decock Society, Edmonton, and the Faculty of Medicine. Equipment supplied by Concept II Inc, Morrissville, VT, USA.

\section{References}

1 Kennedy EJ, editor (1986) Spinal Cord Injury: Facts and Figures. University of Alabama Press, Birmingham, AL.

2 Hoffman MD (1986) Cardiorespiratory fitness and training in quadriplegics and paraplegics. Sports Med 3: 312-330.

3 Shephard RJ, Bouhlel E, Vandewalle H, Monod H (1988) Muscle mass as a factor limiting physical work. $J$ Appl Physiol 64: 1472-1479.

4 Bhambhani YN, Eriksson P, Gomes PS (1991) Transfer effects of endurance training with the arms and legs. Med Sci Sports Exerc 23(9): 1035-1041.

5 Phillips CA, Petrofsky JS, Hendershot DM, Stafford D (1984) Functional electrical exercise-a comprehensive approach for physical conditioning of the spinal cord injured patient. Orthopaedics 7(7): 1112-1123.

6 Petrofsky JS, Brown SW, Cerrel-Bazo H (1992) Active physical therapy and its benefits in rehabilitations. Palaestra 8(3): 23-32.

7 Ragnarsson KT, O'Daniel W Jr, Edgar R, Pollack S, Petrofsky J, Nash MS (1988) Clinical evaluation of computerized functional electrical stimulation after spinal cord injury: a multicenter pilot study. Arch Phys Med Rehabil 69: 672-677.

8 Edwards BG, Marsolais EB (1990) Metabolic responses to arm ergometry and functional neuromuscular stimulation. J Rehabil Res Dev 27(2) 107-114.

9 Davis GM, Servedio FJ, Glaser RM, Gupta SC, Suryaprasad AG (1990) Cardiovascular responses to arm cranking and FNS-induced leg exercise in paraplegics. J Appl Physiol 69(2): 671-677.

10 Hooker SP, Figoni SF, Rodgers MM, Glaser RM, Mathews T, Suryaprasad AG et al (1992) Metabolic and hemodynamic responses to concurrent voluntary arm crank and electrical stimulation exercise in quadriplegics. J Rehabil Res Dev 29(3): 1-11.

11 Figoni SF (1984) Spinal cord injury and maximal aerobic power. Am Corr Ther J 38: 44-50.

12 Ashley EA, Laskin JJ, Burnham R, Steadward RD, Cumming DC, Wheeler GD (submitted for publication) Evidence of autonomic dysreflexia during functional electrical stimulation in spinal cord injured people. To be presented at the 9th International Symposium on Adapted Physical Activity, Yokohama, Japan, August 1993. Paraplegia.

13 Hooker SP, Figoni SF, Glaser RM, Rodgers MM, Ezenwa BN, Faghri PD (1990) Physiologic responses to prolonged electrically stimulated leg cycle exercise in the spinal cord injured. Arch Phys Med Rehabil 71: 863-9.

14 Borg G (1970) Perceived exertion as an indicator of somatic stress. Scand J Rehabil Med 2(3): 92-98.

15 Astrand PO, Rodahl K (1986) Textbook of Work Physiology McGraw-Hill, New York.

16 Wheeler GD, Steadward RD, Stein RB, Weiler M, Belanger M (1993) Functional electrical stimulationassisted strength training for increased muscular strength and bone mass in preparation for standing and walking in spinal cord injured. To be presented at the 9th International Symposium on Adapted Physical Activity, Yokohama, Japan, August 1993. 